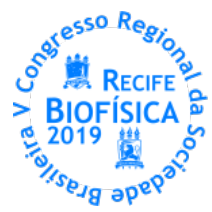

\title{
SUAVE: UMA METODOLOGIA COMPUTACIONAL PARA AVALIAÇÃO DE INTERFACES QUÍMICAS CURVADAS
}

\author{
Denys E. S. Santos ${ }^{1 *}$, Frederico J. S. Pontes ${ }^{1}$, Kaline Coutinho ${ }^{2}$, Roberto D. Lins ${ }^{3}$, Thereza A.Soares ${ }^{1}$, \\ ${ }^{1}$ Departamento de Química Fundamental, UFPE; ${ }^{2}$ Instituto de Física, USP; ${ }^{3}$ Fundação Oswaldo Cruz; \\ *denys.ewerton@ufpe.br
}

\section{INTRODUÇÃO}

A análise de dados é um passo indispensável na engenharia de modelos preditivos para um sistema estudado. 0 sucesso do modelo depende diretamente de quão precisas e, de preferência, eficientes são as análises na medição de propriedades de interesse. O programa SuAVE (Surface Assessment Via grid Evaluation) é um programa baseado em Fortran desenvolvido para analisar as propriedades geométricas das superfícies tendo em conta a morfologia estrutural dos sistemas. Para isso, esse conjunto de rotinas numéricas depende de um processo de montagem de superfície estabelecido com base em geometria diferencial e computacional e cálculo numérico. (DE BERG et al., 2008).

\section{MATERIAIS E MÉTODOS}

O programa foi desenvolvido para lidar com qualquer sistema orgânico, inorgânico ou biológico que contenha uma superfície ou uma interface, apresentando ou não curvatura e exibindo uma ampla gama de morfologias. Pode levar como arquivos de entrada, trajetórias de simulações de dinâmica molecular (MD) e Monte Carlos, bem como qualquer outra metodologia numérica, uma vez que é fornecido um arquivo de entrada no formato PDB (Figura1). O programa pode calcular eficientemente a área e o volume por molécula que compõe uma interface, espessura da membrana, mapas de topologia de superfície, perfis de densidade, parâmetros de ordem de curvatura e curvaturas gaussianas.

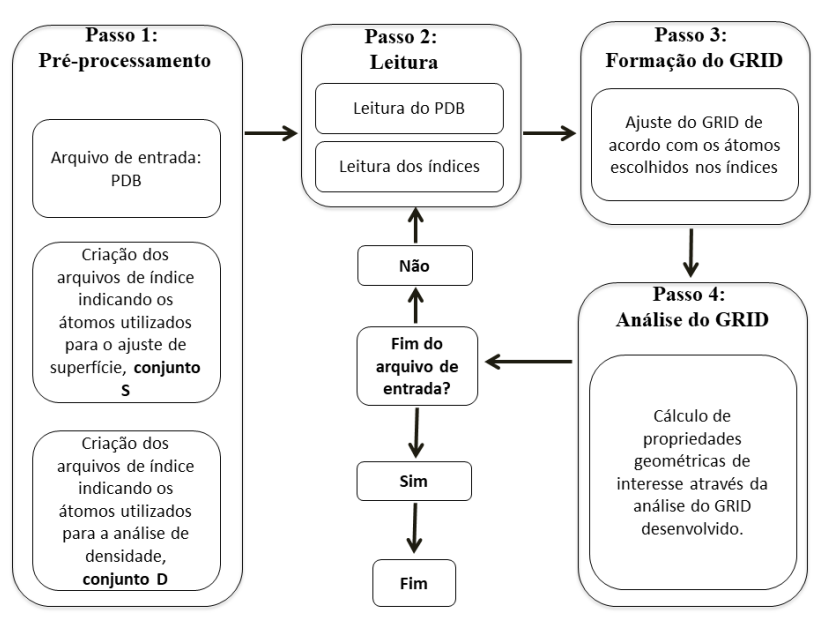

Figura 1: Fluxograma mostrando o funcionamento geral do programa.(fonte: autor)
A precisão da metodologia numérica implementada no SUAVE para avaliar as propriedades geométricas, acima mencionadas, das superfícies curvas, foi validada através da análise de quatro diferentes quimiotipos de Lipídio A e comparada com metodologias convencionais que não levam em consideração a curvatura da membrana. As diferentes bicamadas de Lipídio A apresentam morfologias distintas, como demonstrado pelas simulações de MD (Tabela 1).

Tabela 1: Sistemas simulados. As cores utilizadas nessa tabela serão mantidas durante todo o resumo

\begin{tabular}{cccccc}
\hline Sistemas & Tipo & $\begin{array}{c}\text { Tempo de } \\
\text { simulação }\end{array}$ & Temp. & $\begin{array}{c}\mathbf{N}^{\circ} \text { de } \\
\text { cadeias }\end{array}$ & $\begin{array}{c}\mathbf{N}^{\circ} \text { de } \\
\text { carbonos }\end{array}$ \\
\hline C_H_C14_343 & Curvado & $400 \mathrm{~ns}$ & $343 \mathrm{~K}$ & Hexa & 14 \\
C_T_C10_300 & Curvado & $400 \mathrm{~ns}$ & $300 \mathrm{~K}$ & Tetra & 10 \\
P_H_C10_315 & Plana & $400 \mathrm{~ns}$ & $315 \mathrm{~K}$ & Hexa & 10 \\
P_H_C10_328 & Plana & $400 \mathrm{~ns}$ & $328 \mathrm{~K}$ & Hexa & 10 \\
\hline
\end{tabular}

Todas as caixas de simulação tiveram suas energias minimizadas usando o algoritmo steepest descent sem restrições para 10000 etapas. As caixas de simulação final tinham dimensões ca. $8 \times 12 \times$ $18 \mathrm{~nm}$. Os comprimentos de simulação tiveram um tempo variável, no entanto, um mínimo de 70 ns de trajetória equilibrada foi alcançado para todos os sistemas. As simulações foram realizadas no ensemble isotérmico-isobárica, NPT. O algoritmo Berendsen para pressão e temperatura foi adotado com tempo de acoplamento de 1 ps para pressão e 0,4 ps para temperatura. Um esquema de acoplamento de pressão semi-isotrópico foi adotado usando um valor de 1 bar e compressibilidade de $4,5 \times 10^{-5}$ bar ${ }^{1}$.(BERENDSEN et al., 1984). As equações de movimento foram integradas usando o algoritmo leapfrog e um passo de tempo de 1 fs. Um raio de corte de $1,4 \mathrm{~nm}$ foi utilizado para interações eletrostáticas e van der Waals, além desta distância, as interações eletrostáticas foram tratadas usando o método do campo de reação (do inglês, Reaction Field), juntamente com a constante dielétrica de 66. (TIRONI et al., 1995). As condições de fronteira periódicas foram adotadas através de todas as simulações e não foram utilizadas restrições. Todo o trabalho de construção de caixa e outras simulações foram realizadas usando o pacote GROMACS v.4.5.3. O conjunto de parâmetros adotado nas simulações é uma extensão do campo de força GROMOS. O conjunto de parâmetros de $45 \mathrm{a} 4$ carboidratos foi utilizado para os resíduos de glucosamina e combinado com parâmetros de cadeias de acil disponíveis no 
conjunto de parâmetros lipídicos GROMOS 53A6. (OOSTENBRINK et al., 2004; OOSTENBRINK et al., 2005). 0 modelo de água SPC/E foi utilizado como um solvente explícito. (BERENDSEN, GRIGERA e STRAATSMA, 1987) . O desvio médio dos pontos ajustados com o SUAVE em relação à superfície do sistema foi de $0,11 \mathrm{~nm}$ com um refinamento de grade de 50X50 pontos aplicado a cada análise.

\section{RESULTADOS E DISCUSSÃO}

A comparação da área por grupo de cabeça lipídica pode exemplificar as duas conclusões mais importantes na avaliação sistemática da eficiência de SuAVE (Figura 2 e Figura 3).

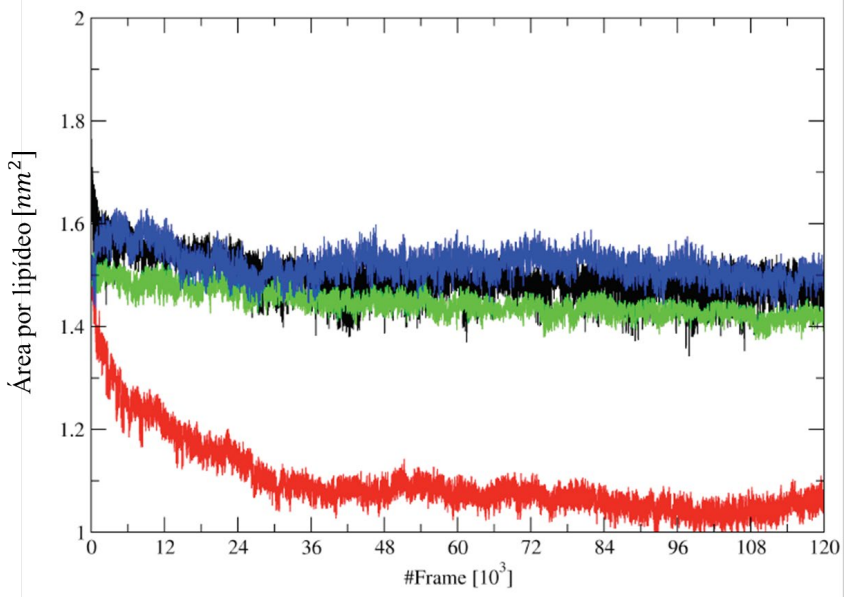

Figura 2: Área por lipídio dos sistemas simulados calculada através do Suave.

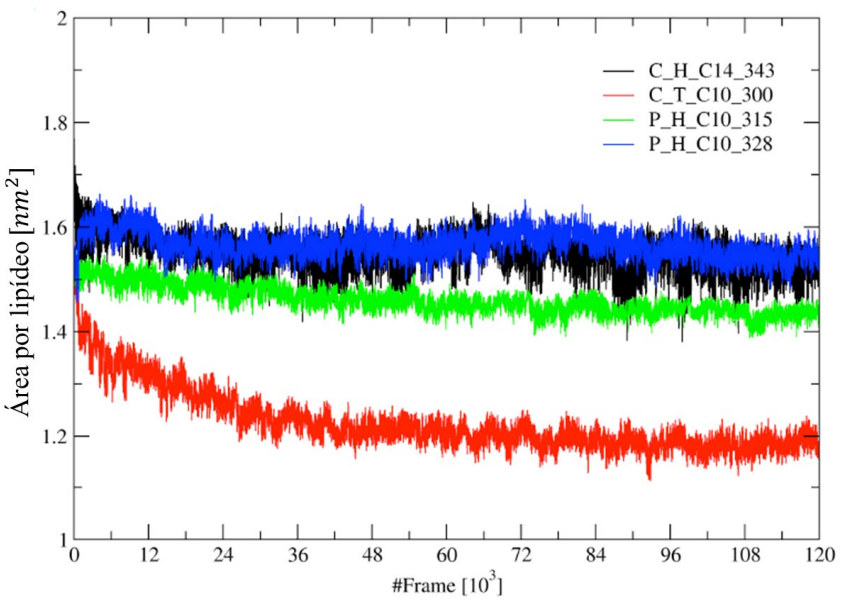

Figura 3: Área por lipídio dos sistemas simulados calculada por uma ferramenta que não leva em consideração a curvatura do sistema.

Para as bicamadas com forma plana lamelar, os valores calculados com SuAVE estão em excelente concordância com a mesma análise realizada com metodologias convencionais. No entanto, ao comparar os resultados das bicamadas na fase ondulatória, a análise convencional subestima a área por molécula lipídica em cerca de $11,57 \%$ porque negligencia a curvatura da membrana. Do mesmo modo, os perfis de densidade para as bicamadas que apresentam curvatura em sua morfologia são bastante distintos quando a curvatura da membrana é levada em consideração, enquanto eles são idênticos para as bicamadas em fase lamelar que não apresentem curvatura elevada em sua morfologia (Figura 4).

Os perfis de densidade calculados com o SUAVE para os sistemas anteriores mostram que, mesmo não sendo planos, os sistemas com curvatura eles conservam uma estrutura lamelar. Por outro lado, os perfis de densidade obtidos através do uso de análises convencionais implicam na presença de água dentro da membrana,
- que conduz claramente a uma interpretação equivocada dos dados. Além disso, o SuAVE calcula o parâmetro de ordem de curvatura que fornece uma estimativa quantitativa de quanto, em média, é o valor da curvatura de uma determinada superfície. A apresentação desse resultado é obtida pelo gráfico com distintas tonalidades para cada valor de curvatura. É interessante observar como essa análise consegue demarcar as regiões onde há presença de curvatura, positiva ou negativa, na interface. Essa mesma análise foi utilizada por Santos et al. (2017) para avaliar, satisfatoriamente, a modificação estrutural da membrana externa de uma $P$. aeruginosa ao entrar em contato com peptídeos antimicrobianos. Ao usar os ângulos de curvatura da superfície em relação ao eixo normal do sistema, é possível demonstrar que, para os sistemas planares, cerca de $90 \%$ da superfície exibe um ângulo de curvatura da superfície inferior a $30^{\circ}$, enquanto que para as membranas curvadas, esse valor aumenta para $60^{\circ}$, estando em total acordo com o que seria esperado (Figura 5 e Figura 6).
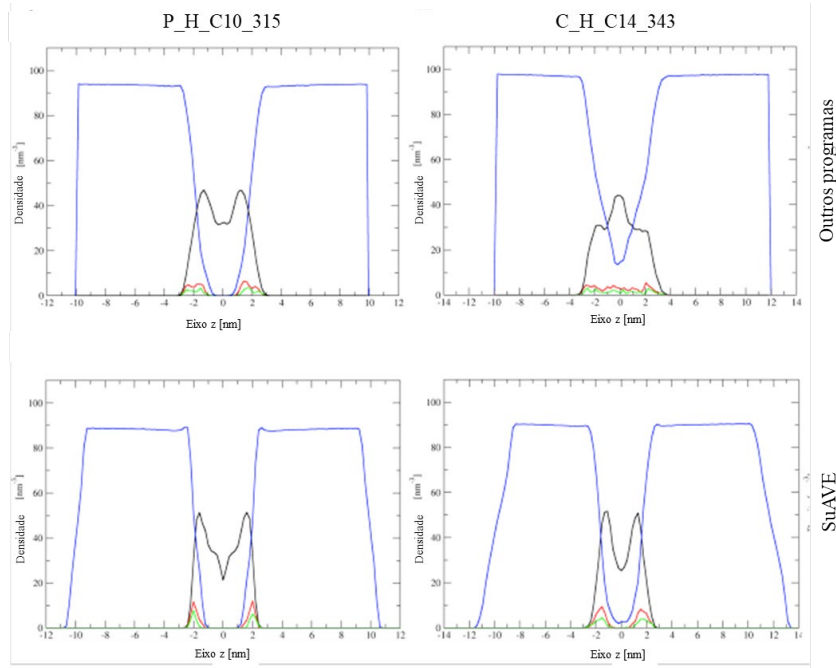

Figura 4: Perfis de densidade para u=dois dos sistemas simulados utilizando SUAVE e uma ferramenta convencional que não leva em consideracão a curvatura da superfície das membranas. Água em azul, cadeias alquílicas em preto, contra-íons em verde e fosfatos em vermelho.
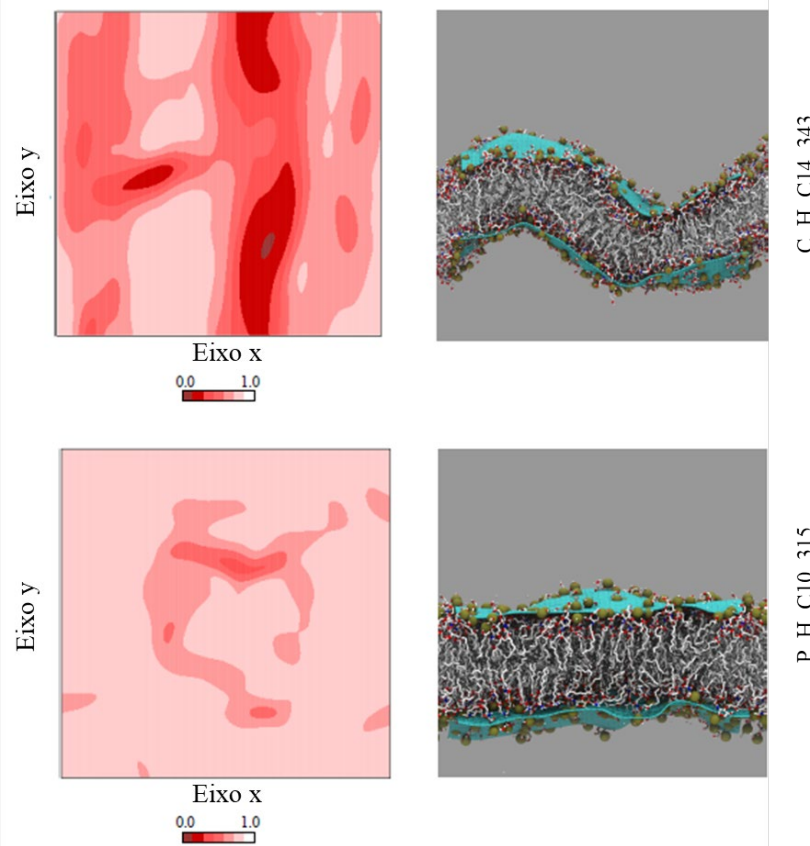

Figura 5: Parâmetro de ordem de curvatura para dois dos sistemas simulados, lado esquerdo. Estrutura final das membranas, lado direito. 


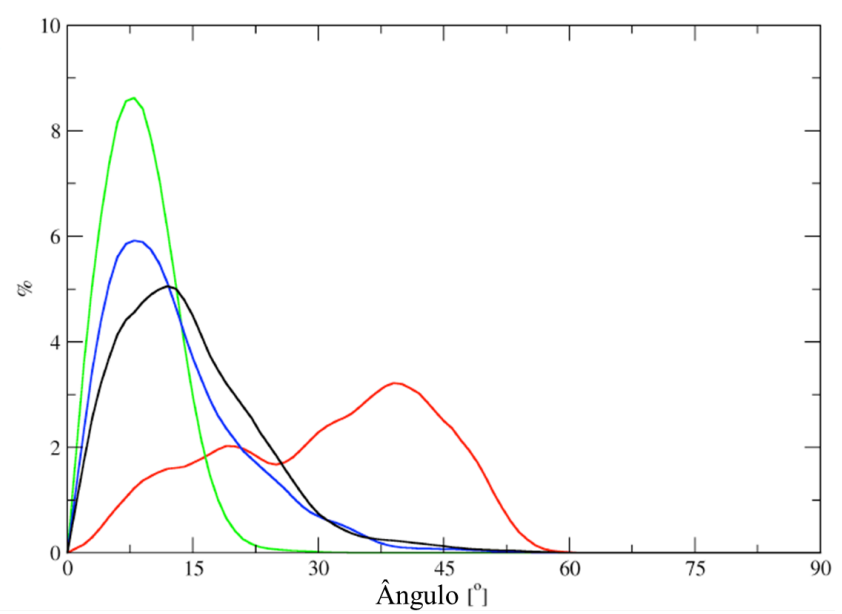

Figura 6: Distribuição dos ângulos de curvatura médios para cada um dos sistemas dos sistemas estudados. Em preto está o sistema C_T_C10_300, em vermelho o sistema C_H_C14_343, em verde está o P_H_C10_315 e em azul o sistema P_H_C10_328.

\section{CONCLUSÕES}

Através de todos esses exemplos, o SuAVE demonstrou capacidades para avaliar propriedades estruturais de diferentes sistemas, levando em consideração sua geometria constitucional, não observada na análise numérica convencional, mostrando potencial para contribuir na avaliação de uma gama mais ampla de sistemas orgânicos, inorgânicos ou biológicos.

\section{REFERÊNCIAS}

BERENDSEN, H. J. C.; POSTMA, J. P. M.; VAN GUNSTEREN, W. F.; DINOLA, A.; HAAK, J. R. Molecular dynamics with coupling to an external bath. J. Chem. Phys., v. 81, p. 3684-3690, 1984.

BERENDSEN, H. J. C.; GRIGERA, J. R.; STRAATSMA, T. P. The missing term in effective pair potentials. J. Phys. Chem., v. 91(24), p. 6269-6271, 1987.

DE BERG, M.; CHEONG, O.; VAN KREVELD, M.; OVERMARS, M. Computational Geometry. Algorithms and Applications. Springer 2008.

OOSTENBRINK, C.; VILLA, A.; MARK, A. E.; VAN GUNSTEREN, W. F. A biomolecular force field based on the free enthalpy of hydration and solvation: the GROMOS force-field parameter sets 53A5 and 53A6. J. Comp. Chem., v. 25(13), p. 1656- 1676, 2004.

OOSTENBRINK, C.; SOARES, T. A.; VEGT, N. F. A.; VAN GUNSTEREN, W. F. Validation of the $53 A 6$ GROMOS force field. European Biophysical Journal, v. 34, p. 273-284, 2005.

SANTOS, D. E. S.; POL-FACHIN, L.; LINS, R. D.; SOARES, T. A. J. Polymyxin Binding to the Bacterial Outer Membrane Reveals Cation Displacement and Increasing Membrane Curvature in Susceptible but Not in Resistant Lipopolysaccharide Chemotypes. J. Chem. Inf. Model., v. 57, p. 2181-2193, 2017.

TIRONI, I. G.; SPERB, R.; SMITH, P. E.; VAN GUNSTEREN, W. F. A generalized reaction field method for molecular dynamics simulations. J. Chem. Phys., v. 102, p. 5451-5459, 1995. 